\title{
FAST PHOTOPROCESSES IN A SYMMETRIC INDOTRICARBOCYANINE DYE (HITC) IN SOLUTIONS
}

\author{
M. P. Samtsov, ${ }^{a^{*}}$ S. A. Tikhomirov, ${ }^{\text {b }}$ O. V. Buganov, ${ }^{b}$

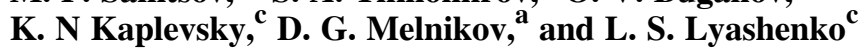

UDC 535.34

Spectral-kinetic and photochemical properties of HITC dye with iodide and perchlorate counterions have been studied in environments where the dye molecules exist in different ionic forms. In ethanol, the dye molecules exist as free ions; in dichlorobenzene, as contact ion pairs. Superfast transformation of non-stationary spectra in an HITC dye bleaching band is found. The observed effects are interpreted within the framework of concepts on "burning out" a notch in the contour of a non-uniformly widened vibronic band of $S_{0} \rightarrow S_{1}$-absorption. Qualitative differences in recorded absorption spectra from the dye excited electronic states for weakly and highly polar solvents are found. It is shown that the observed differences are caused by superfast charge transfer in the contact ion pairs that results in the formation of free radicals.

Key words: indotricarbocyanine dyes, ion pairs, spectral properties, free radicals.

Introduction. One possible application of cationic polymethine dyes is their use as photosensitizers for cancer photochemotherapy $[1,2]$. The mechanism of the photoactivity of this class of compounds does not fit into the framework of a scheme in which the tumor cells are damaged exclusively by the formation of photosensitizer molecules of singlet oxygen $[1,2]$. Results of in vitro research on the photocytotoxicity of polymethine dyes on cellular structures $[3,4]$ led to the conclusion that the most probable mechanism of damage to cancer cells was the generation of highly reactive radicals by excited dye molecules. Photo-induced formation of free radicals that occurs as a result of phototransfer of charge in contact ion pairs has been observed and studied for compounds of the polymethine dye class only for dyes with exceedingly specific bulky counter-ions such as triphenylbutylborate [5]. The conclusion about charge transfer for such compounds was made based on an analysis of results on the formation of the final product assuming the existence of a complicated chain of radical reactions initiated by primary electron transfer in the ion pair. There is practically no mention in the literature of direct detection of photo-induced charge transfer in ion pairs of indotricarbocyanine dyes containing other anions typical of polymethine dyes. Furthermore, there are contradictory opinions about the ability of photochemical reactions associated with electron phototransfer in contact ion pairs of polymethine dyes with simple "inert" ions such as $\mathrm{I}^{-}$and $\mathrm{ClO}_{4}^{-}$to occur because of the high oxidation potentials of these ions compared with triphenylbutylborate [5].

Indotricarbocyanine dyes are localized in cancer cells in the region with low dielectric permeability and are found primarily as contact ion pairs [6]. The last circumstance in addition to data on the retention of the photocytotoxicity of these compounds under hypoxic conditions [1] and previous results [3,4] allows the initial damage mechanism to cancer cells to assume photo-induced charge transfer in contact pairs that leads to the formation of free radicals. Cationic polymethine dyes in solutions can be found as a mixture of several types of ionic species such as free ions and contact ion pairs and ion pairs separated by solvent [7-9]. The equilibrium between these species shifts to one side or another upon changing the nature of the solvent or the temperature, replacing the counter ion, or adding to the solution ionic and solvating additives [10].

\footnotetext{
*To whom correspondence should be addressed.
}

${ }^{a}$ A. N. Sevchenko Research Institute for Applied Physical Problems, 7 Kurchatov St., Minsk, 220064, Belarus; e-mail: samtsov@bsu.by; ${ }^{b}$ B. I. Stepanov Institute of Physics, National Academy of Sciences of Belarus, Minsk; e-mail: tikhomirov@imaph.bas-net.by; ${ }^{\mathrm{c}}$ Belarussian State University, Minsk. Translated from Zhurnal Prikladnoi Spektroskopii, Vol. 76, No. 6, pp. 830-838, November-December, 2009. Original article submitted May 20, 2009. 
Herein we present results from studies of the spectral-kinetic and photochemical properties of HITC dye with iodide and perchlorate counter ions in which the dye molecules are found in various ionic forms. Femtosecond kinetic spectroscopy methods were used to study the dynamics of non-stationary absorption spectra of solutions while maintaining conditions that ensure conversion of indotricarbocyanine dye molecules in a certain ionic form into the excited state. The main goal of the work was to observe possible fast charge transfer in the contact ion pairs of indotricarbocyanine dyes with simple anions and to analyze the contribution of other possible superfast relaxation processes to the dynamics of the recorded non-stationary spectra.

Experimental. The indotricarbocyanine dye HITC with perchlorate $\left(\mathrm{ClO}_{4}^{-}\right)$and iodide $\left(\mathrm{I}^{-}\right)$counter ions was selected for the studies:

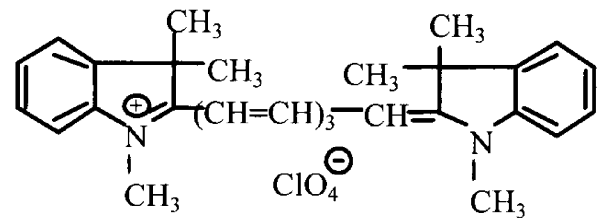

The purity of the dye was checked by HPLC analysis. In addition, the position and shape of the fluorescence spectra of their ethanol solutions were confirmed to be independent of the excitation wavelength; the fluorescence excitation spectra, of the detection wavelength.

We used mainly ethanol and dichlorobenzene (DCB) as solvents. These were purified by the standard methods [11]. Solvents were selected partially because the absorption spectra of the studied dyes in weakly polar DCB have the same position as their spectra in cellular structures [6]. Another important consideration for selecting the solvents was their identical viscosities at room temperature. This was important because the solvent viscosity has a significant effect on the probability of intramolecular dissipation of electron-excitation energy in the polymethine dyes [12, 13]. Several studies have been carried out in deuterated $\mathrm{CHCl}_{3}$ and deuterated $\mathrm{CH}_{3} \mathrm{CN}$ without additional purification.

Fluorescence and excitation fluorescence spectra and the degree of fluorescence polarization were recorded using a Fluorolog (Spex) spectrofluorimeter; electronic absorption spectra, PV 1251A (Solar) or Specord M40 spectrophotometers. The dye fluorescence decay kinetics were analyzed in the nanosecond range using a pulsed spectrofluorometer developed in the Laboratory of Spectroscopy at the Research Institute for Applied Physical Problems [14]. Samples were held at constant temperature because the luminescence characteristics of the polymethine dyes depended significantly on the temperature and solution viscosity. The dye photodestruction quantum yield $(\Phi)$ was determined by the literature method [15]. The quantum yield $(B)$ for generation of singlet oxygen $\left({ }^{1} \mathrm{O}_{2}\right)$ by the studied compounds was determined based on the quantum yield measured by a relative method of dye-sensitized luminescence of ${ }^{1} \mathrm{O}_{2}\left(\Phi_{\text {lum }}\right)$ and the ratio [16] $\Phi_{\text {lum }}=B \tau_{0} / \tau_{\mathrm{p}}$, where $\tau_{0}$ is the lifetime; $\tau_{\mathrm{p}}$, the radiation lifetime of singlet oxygen. The dye HITCI was used as a standard. The quantity $B_{\mathrm{e}}=3.6 \%$ for it in deuterated $\mathrm{CHCl}_{3}$ [17]. The lifetime of singlet oxygen and the radiation lifetime of ${ }^{1} \mathrm{O}_{2}$ for the used solvents were taken from the literature [18, 19].

Non-stationary absorption spectra were recorded using a femtosecond spectrometer [20] based on an original Ti:Sp generator of femtosecond pulses [21] that was pumped synchronously by a Nd:YAG pulse laser with passive mode synchronization and negative feedback. This system can produce at $10 \mathrm{~Hz}$ retuned pulses in the spectral range $760-820 \mathrm{~nm}$ of duration $\sim 150 \mathrm{fsec}$ and $\leq 1 \mathrm{~mJ}$ energy. Pulses of the main frequency that were obtained at the amplifier output were divided into two parts with a 1:4 ratio of intensities. The more intense beam was used as the pump pulse after passing the regulated delay line. Changes of optical density of the studied samples were recorded using a supercontinuum generated in a $1-\mathrm{cm}$ cuvette with distilled water by focusing on it the smaller part of the main frequency pulse. The resulting continuum and the spectral sensitivity of the photodetector enabled a probe signal to be formed in the spectral range 350-1000 $\mathrm{nm}$. Continuum emission was divided by a semi-transparent mirror into two pulses of identical intensity (reference and signal) that were focused on the sample using an optical mirror. The reference pulse passed through the cuvette with the studied compound before applying the excitation pulse. The signal pulse passed through the excited volume with a certain delay relative to the excitation pulse. Spectra of both pulses for each laser flash were recorded by a system based on a polychromator and a CCD-matrix with subsequent data processing by a microprocessor. The half-width of the cross-correlation function was $\sim 350$ fsec for all probe wave- 
TABLE 1. Spectral Characteristics of HITC Dye with Various Anions in Solutions

\begin{tabular}{|c|c|c|c|c|c|c|c|c|}
\hline Solvent & $\begin{array}{c}\lambda_{\max }^{\mathrm{abs}}, \\
\mathrm{nm}\end{array}$ & $\begin{array}{c}\Delta \lambda_{1 / 2}^{\mathrm{abs}} \\
\mathrm{nm}\end{array}$ & $\begin{array}{c}\lambda_{\max }^{\mathrm{fl}}, \\
\mathrm{nm}\end{array}$ & $\begin{array}{c}\Delta \lambda_{1 / 2}^{\mathrm{fl}}, \\
\mathrm{nm}\end{array}$ & $\begin{array}{c}\tau, \\
\text { nsec }\end{array}$ & $\varphi_{\mathrm{fl}}$ & $\Phi$ & $B, \%$ \\
\hline \multicolumn{9}{|c|}{$\mathrm{ClO}_{4}^{-}$} \\
\hline$d$-Chloroform & 755 & 60 & 785 & 49 & 1.8 & 0.36 & $3.1 \cdot 10^{-4}$ & 0.5 \\
\hline Dichlorobenzene & 766 & 49 & 796 & 46 & 1.6 & 0.42 & $1.3 \cdot 10^{-6}$ & - \\
\hline Ethanol & 742 & 53 & 773 & 48 & 1.4 & 0.28 & $4.6 \cdot 10^{-7}$ & - \\
\hline$d$-Acetonitrile & 739 & 58 & 771 & 47 & - & 0.43 & $3.0 \cdot 10^{-5}$ & 0.2 \\
\hline \multicolumn{9}{|c|}{$\mathrm{I}^{-}$} \\
\hline$d$-Chloroform & 755 & 63 & 785 & 49 & 1.8 & 0.36 & $1.1 \cdot 10^{-3}$ & 3.6 \\
\hline Dichlorobenzene & 766 & 49 & 796 & 46 & 1.6 & 0.42 & $3.1 \cdot 10^{-5}$ & - \\
\hline Ethanol & 742 & 53 & 773 & 48 & 1.4 & 0.28 & $4.7 \cdot 10^{-7}$ & - \\
\hline$d$-Acetonitrile & 739 & 58 & 771 & 47 & - & 0.43 & $3.0 \cdot 10^{-5}$ & 0.2 \\
\hline
\end{tabular}

lengths. The minimum recorded change of optical density for each delay line position with signal averaging over 100 pulses was $\approx 0.5 \cdot 10^{-3}$.

The dye concentration in samples used for studies with the femtosecond spectrometer was $(0.4-1.0) \cdot 10^{-5} \mathrm{M}$. Measurements were made in 2-mm cuvettes. The same sample optical density at the excitation wavelength was chosen in order to ensure similar measurement conditions for different solvents. The energy of the exciting pulses was $\approx 200 \mu \mathrm{J}$ with pump beam diameter in the overlap region of the exciting and probe pulses of $6 \mathrm{~mm}$. Band intensities and the shapes of stationary sample absorption spectra did not differ before the start and after the completion of series of measurements. This indicated that the studied compounds were photostable.

Results and Discussion. As noted above, an actual combination of conditions under which the dye in solutions was found in a certain ionic form was required for each polymethine dye. The molecular state of the dyes in various solvents was studied using stationary spectroscopy methods in order to select such conditions.

Obviously data allowing the absence or presence of a layer of solvent molecules between the anion and dye cation to be established, i.e., to give information about the distance between them, were most convincing regarding the presence in the solution of contact ion pairs of dye molecules. An increased probability of intersystem crossing as a result of the heavy-atom effect could be expected if heavy atoms were present in the dye anion and its molecules existed in solution as contact ion pairs [22]. In fact, the yield of molecules in the triplet state increased by more than 30 times if the $\mathrm{H}$ was replaced by $\mathrm{I}$ in the chain mesoposition for symmetric cationic polymethine dyes [23]. Also, substitution of $\mathrm{F}$ by $\mathrm{I}$ in the cation terminal groups increased the yield of molecules in the triplet state by $\sim 3$ times. This demonstrated the extent of the effect of the position of the heavy atoms in the polymethine chain on the rate of intersystem crossing. Thus, it should be expected that the heavy-atom effect in dilute solutions, where the effect of neighboring dye molecules is excluded, should affect the yield of molecules in the triplet state only for contact ion pairs (anion located close to the cation). Therefore, the efficiencies of their transitions into the triplet state were compared for molecules with heavy $\left(\mathrm{I}^{-}\right)$and light $\left(\mathrm{ClO}_{4}^{-}\right)$anions. The results were used as an indicator of the state of ionic equilibrium in the solution.

The shape and position of the absorption and fluorescence spectra were the same for perchlorate and iodide dyes in highly polar ethanol and deuterated $\mathrm{CH}_{3} \mathrm{CN}$ (Fig. 1). The quantum yields and fluorescence life-times were also identical (Table 1). Furthermore, the photodestruction quantum yields coincided for the studied dye pair in ethanol and deuterated $\mathrm{CH}_{3} \mathrm{CN}$ at a concentration of $\sim 10^{-6} \mathrm{M}$. The quantum yields of singlet oxygen luminescence that was sensitized by these dyes were also identical in deuterated $\mathrm{CH}_{3} \mathrm{CN}$, for which the lifetimes and efficiencies of dissolved oxygen emission are typically rather large. The values of the parameters $B$ and $\Phi$ determine the yield of dye molecules in the triplet state because the main mechanism of singlet oxygen sensitization by the studied molecules in solutions is radiationless energy transfer from the dye molecules in the triplet state; the photodestruction mechanism, self-sensitized oxidation by single oxygen [24]. Therefore, the agreement of these parameters for iodide and per- 


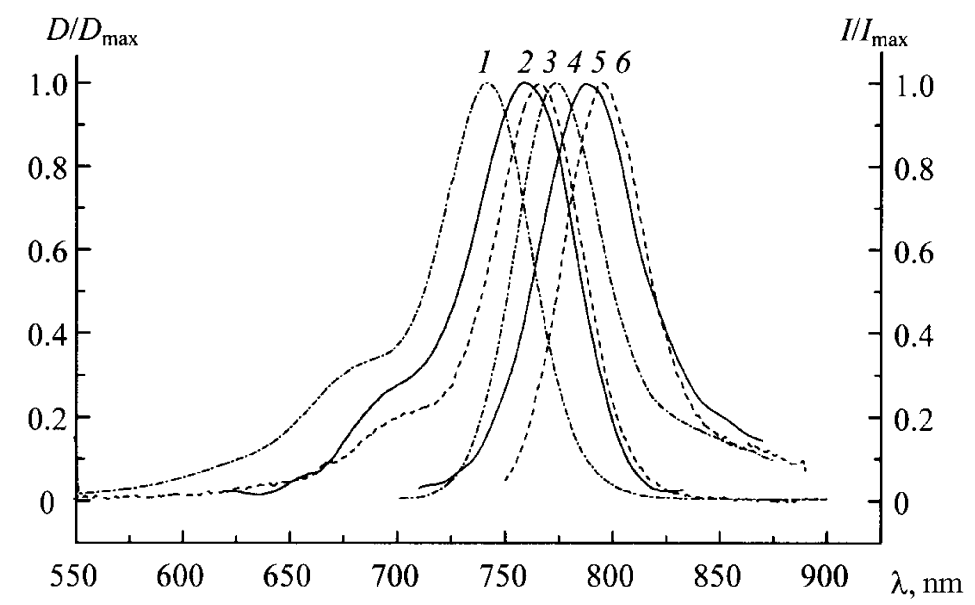

Fig. 1. Absorption (1-3) and fluorescence (4-6) spectra of dye HITC in ethanol $(1,4)$, dichlorobenzene $(3,5)$, and HeLa cells $(2,6)$.

chlorate dyes indicated that the studied compounds had identical yields into the triplet state in polar solvents and that the dye cations were at a distance from the anions, i.e., the compounds were found as free ions. The agreement of the spectral-luminescence characteristics of these dyes in highly polar solvents also indicated that the molecules were dissociated in solution.

The shape and position of the absorption and fluorescence spectra coincided (Fig. 1) and the quantum yields and fluorescence lifetimes were identical (Table 1) for both dyes in weakly polar DCB. However, the photodestruction quantum yields differed considerably in DCB, in contrast with the highly polar solvents. Thus, the photodestruction quantum yield of the iodide was two times greater than that of the perchlorate at identical dye concentrations $\left(\sim 10^{-6} \mathrm{M}\right)$. The absorption and fluorescence spectra of the studied dyes in deuterated $\mathrm{CHCl}_{3}$ also had practically the same shape and position. Their quantum yields and lifetimes coincided. Furthermore, the photodestruction quantum yield for the iodide dye was more than an order of magnitude greater compared with that of the perchlorate. In addition, the quantum yield for singlet oxygen generation in deuterated $\mathrm{CHCl}_{3}$ was also an order of magnitude greater for the iodide than for the perchlorate. Considering the low solution concentrations $\left(\sim 10^{-6} \mathrm{M}\right)$, the results indicated that the efficiency of intersystem crossing was much higher for the iodide than the perchlorate dye in weakly polar solvents. Therefore, the heavy-atom effect was evident in weakly polar solvents. This was possible only if the anion and the dye-cation polymethine chain were rather close. The results led to the conclusion that the studied compounds in DCB and deuterated $\mathrm{CHCl}_{3}$ existed as contact ion pairs. The small half-width of the dye absorption spectra in DCB, the agreement of the positions and shapes of their fluorescence spectra upon changing the excitation wavelength, and the monoexponential fluorescence decay kinetics were consistent with the presence in solution of only one type of emitting center.

Based on the results, we selected DCB and ethanol as the solvents for further measurements by femtosecond spectroscopy. The compounds in these solvents were found in strictly defined ionic states. The characteristics of the samples did not change during series of measurements. They had practically the same viscosity at room temperature.

Figure 2 shows differential non-stationary perchlorate dye absorption spectra in DCB that were corrected for group rate dispersion for three delay times $\Delta t$ between the exciting and probe pulses. The non-stationary spectra of the dye iodide were practically identical to those shown. An induced $S_{1} \rightarrow S_{n}$-absorption appeared in the visible region. A negative signal (bleaching of the $S_{0} \rightarrow S_{1}$-absorption band and strengthening of the fluorescence band) was found in the near-IR region. Distortions due to incidence on the photodetector of pump radiation that was not transformed into the continuum appeared near the maximum of the main harmonic of the Ti:Sp laser $\left(\lambda_{\mathrm{ex}} \sim 775 \mathrm{~nm}\right)$ in the nonstationary spectra. The spectra also had a common shape for dye solutions in polar ethanol. It is noteworthy that the common shape of the resulting spectra was similar to that of published spectra [25] for indotricarbocyanine dyes of similar structure. 


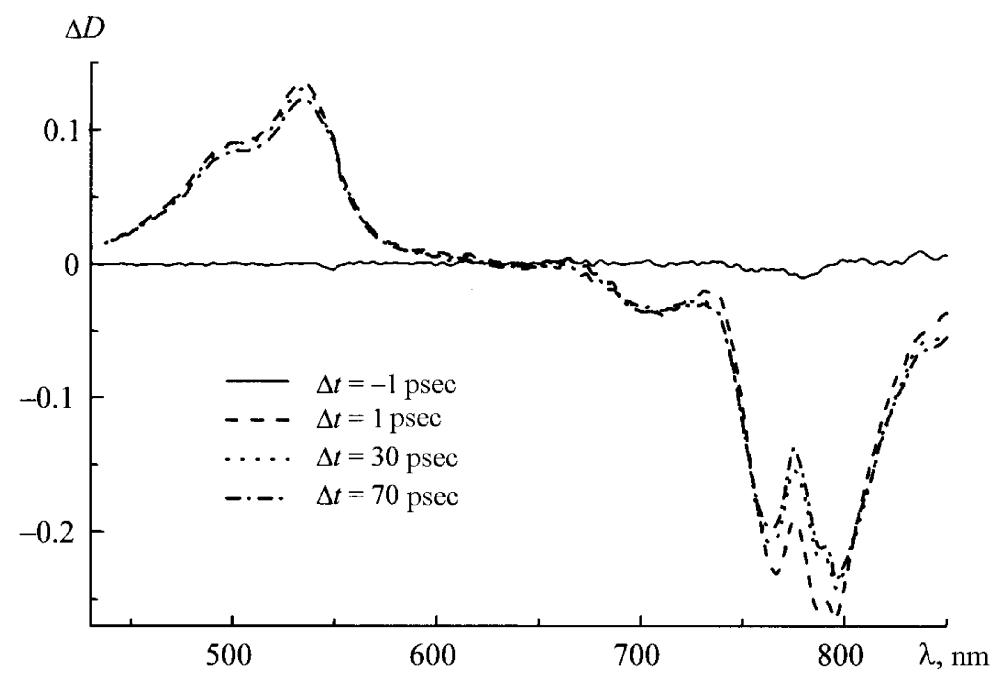

Fig. 2. Differential spectra of non-stationary absorption of dye HITC in dichlorobenzene at various delay times.

As it turned out, the shape of the non-stationary spectra did not substantially change within the limits of the studied time regime (up to $150 \mathrm{psec}$ ). An overall weak signal decay was observed in induced absorption and amplification bands due most likely to relaxation of the excited singlet state, the lifetime of which for the studied compound in DCB was $1.6 \mathrm{nsec}$. Also, a delay in the in-growth of the amplification with a characteristic time 4 psec was recorded in the amplification band for the DCB and ethanol solutions. This agreed with the reported data [26] for an ethanol solution of HITC iodide $(3.5 \mathrm{psec})$ that were interpreted as the manifestation of vibrational relaxation in the excited electronic state that was due to intermolecular interactions.

The envelope of the bleaching band $(650-800 \mathrm{~nm})$ underwent small characteristic transformations in the time range $\leq 30 \mathrm{psec}$. These consisted of the additional in-growth of a signal on the short-wavelength tail and a corresponding decay of the signal in the spectral region close to the exciting radiation. An isosbestic point was observed at $\sim 755 \mathrm{~nm}$. Spectral transformations in the bleaching band appeared analogously for the ethanol solution. The recorded changes in the bleaching band envelope suggested that excitation of solutions of the studied compound by femtosecond pulses caused "burning out" of a notch in the envelope of a nonuniformly broadened vibronic absorption band and its subsequent "quenching" as a result of redistribution of vibrational energy through intermolecular interactions. Figure 3 shows the optical density kinetics in the characteristic spectral ranges. The calculated and experimental functions agreed best within the framework of the proposed model in DCB and ethanol for characteristic intermolecular vibrational relaxation times $\tau_{\mathrm{vib}} \sim 10$ psec. A short (several hundred femtoseconds) component was recorded for all kinetics. This might have been due either to intramolecular vibrational relaxation similar to that previously reported [26] or to the appearance of coherent effects in the overlap region of the exciting and probe pulses. It should be noted that the times for intermolecular redistribution of vibrational energy (thermalization) for the ground electronic state of the studied molecule turned out to be much greater than the analogous characteristics for the excited electronic state. Thus, the analysis of the bleaching band transformation immediately after the action of the exciting pulse made it possible to determine the characteristic times of intermolecular thermalization in the ground electronic state of the studied molecule.

Figure 4 shows absorption spectra from excited states of the studied compound in DCB and ethanol that were normalized for excitation (recalculated for the identical amount of excited centers). The conversion from differential spectra $(\Delta D)$ to absorption spectra $(\varepsilon)$ from the excited state was made using the standard method [4]. In contrast with the literature [4], the shape of the absorption spectrum from the excited electronic state was analyzed for time delays $>30 \mathrm{psec}$ in order to exclude effects associated with vibrational non-equilibrium.

Stationary absorption spectra of the dye in DCB and ethanol had similar shapes. The only difference was that spectra in DCB compared to those in ethanol were shifted by $14 \mathrm{~nm}$ to the long-wavelength region (corresponding to 


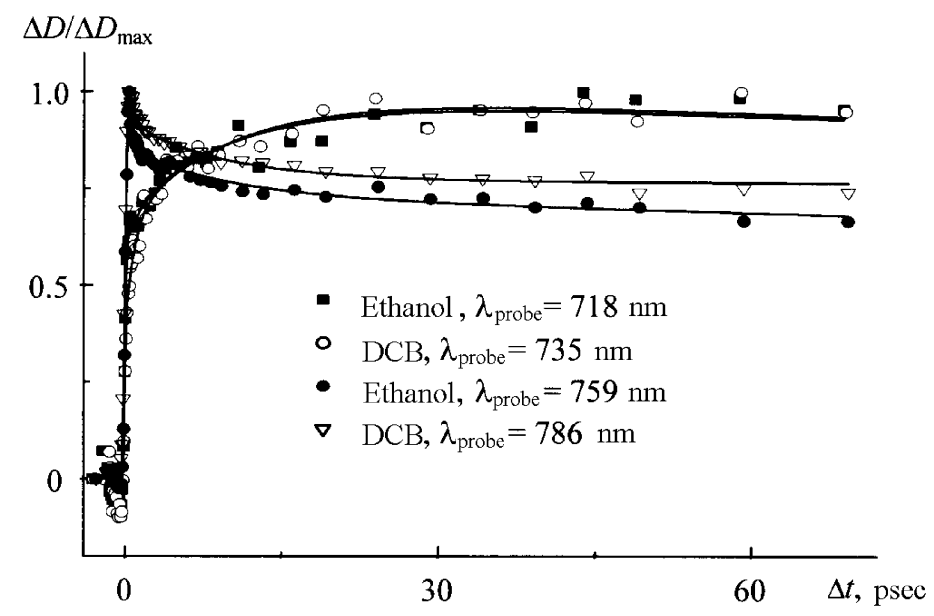

Fig. 3. Kinetics of induced optical density in the bleaching band of dye HITC solutions.

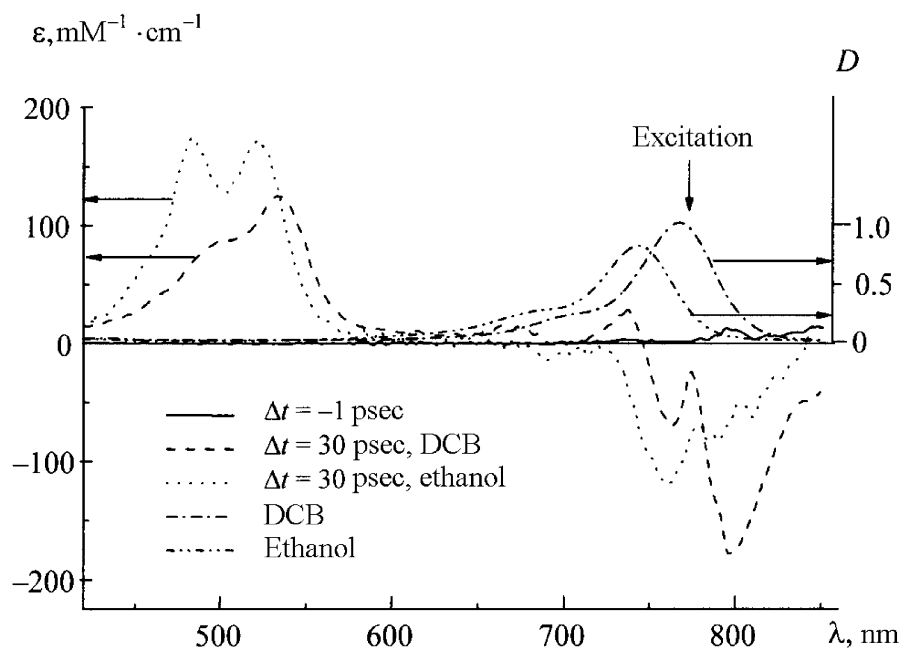

Fig. 4. Absorption spectra normalized for excitation from the excited state and stationary spectra of dye HITC solutions in dichlorobenzene and ethanol.

$\left.422 \mathrm{~cm}^{-1}\right)$ and had a 4-nm smaller half-width. Dye absorption spectra in the short-wavelength region $(300-450 \mathrm{~nm})$, which corresponded to transitions into higher energy electronic states, also exhibited a bathochromic shift of the bands by $\sim 440 \mathrm{~cm}^{-1}$. Figure 4 shows that absorption spectra from the excited electronic state were substantially different for the studied solvents. For the DCB solution, the shape of the induced absorption band changed in the visible spectral region due to $S_{1} \rightarrow S_{n}$-absorption of the molecules and an additional band appeared in the near-IR region at $\lambda_{\max }=$ $736 \mathrm{~nm}$. As noted above, the observed differences in non-stationary absorption spectra can be related to the formation of free radicals as a result of superfast charge transfer in contact ion pairs because the molecules in DCB as contact ion pairs and in ethanol as free ions transition into the excited state upon excitation of the studied dye solution. The recorded non-stationary spectrum of the dye in DCB in this instance is a superposition of the absorption spectrum from the excited electronic state of neutral molecules and that of free radicals formed practically without a time delay.

The fast radical formation rate $\left(k \geq 0.6 \cdot 10^{13} \mathrm{sec}^{-1}\right)$ with a rather long lifetime for the $S_{1}$-state of the studied compound (1.6 nsec) can be justified assuming that charge transfer occurs either from higher electronic states $S_{n}$ or through a non-thermalized Franck-Condon state $S_{1}^{*}$. However, excitation in our instance occurred in the long-wave- 
length tail of the main absorption band so that such an explanation is unsuitable in principle. Also, the observed effects can be interpreted assuming that electron phototransfer from the $\mathrm{ClO}_{4}^{-}$anion to the polymethine dye cation $\mathrm{PD}^{+}$ occurs with formation of free radicals only for a certain part of contact pairs (closest) practically without a delay and during the effective time of the exciting pulse.

This interpretation was based on the assumption that contact pairs formed by the same type of ions can have different distances between the ions and similar spectral properties. In fact, it has been shown [27] that ion pairs of the same type can have different average distances between ions depending on the solvent although the spectral properties differ insignificantly. Obviously, a dynamic equilibrium of contact ion pairs with different distances between ions exists in an actual solution. Such pairs have similar spectral characteristics and can differ radically with respect to the ability for electron phototransfer. Phototransfer can occur with a high rate constant only for excitation of close contact ion pairs. The estimated formation time of the induced absorption band due to free radicals with a maximum at $736 \mathrm{~nm}(\leq 150 \mathrm{fsec})$ is consistent with a high electron-transfer rate $\left(k \geq 0.6 \cdot 10^{13} \mathrm{sec}^{-1}\right)$. Obviously, charge phototransfer for such close contact pairs is the main channel for dissipating excitation energy. Also, fast charge phototransfer is hindered for ion pairs with a large distance between the ions. The experimentally recorded fluorescence kinetics of ion pairs with $\tau_{\mathrm{fl}}=1.6 \mathrm{nsec}$ characterizes just such centers. It is noteworthy in this respect that the concept of penetrating ion pairs in which the volume of the ion pair is much less than the sum of the volumes of its component ions was introduced for polymethine dyes with specific bulky counter ions in order to interpret results for charge phototransfer in contact ion pairs [5].

Thus, superfast (within the limits of the exciting pulse) formation of free radicals together with the known lifetime of the excited singlet state of the neutral molecules made it possible to propose that charge transfer occurred only for contact pairs that can be defined as the closest contact ion pairs.

Conclusion. Stationary and high-speed spectroscopy methods were used to study spectral-kinetic and photochemical properties of HITC dye with iodide and perchlorate counter ions in solutions of various polarity. It was found that the dyes in ethanol are found as free ions; in DCB, as contact ion pairs. Superfast transformation of nonstationary spectra in the HITC dye bleaching band was observed. The observed effects were interpreted using concepts of burning out of a notch in the envelope of a nonuniformly broadened vibronic $S_{0} \rightarrow S_{1}$-absorption band. Characteristic times for vibrational relaxation in the ground electronic state were estimated. Qualitative differences in the recorded absorption spectra from excited dye electronic states were observed for weakly and highly polar solvents. It was shown that the observed differences could be explained by superfast charge transfer in contact ion pairs, as a result of which free radicals were formed. Superfast electron transfer in HITC contact pairs was identified spectrally and was accompanied by formation of free radicals. This process could form the basis of the mechanism responsible for the photocytotoxicity of symmetric indotricarbocyanine dyes under hypoxic conditions.

\section{REFERENCES}

1. Y. P. Istomin, E. N. Alexandrova, E. A. Zhavrid, E. S. Voropay, M. P. Samtsov, K. N. Kaplevsky, A. P. Lugovsky, and A. A. Lugosky, Exp. Oncol., 28, 80-82 (2006).

2. E. Delaey, F. Laar, D. Vos, A. Kamuhabwa, P. Jacobs, and P. Witte, J. Photochem. Photobiol. B, 55, 27-36 (2000).

3. O. Valdes-Aguilera, G. Ara, and E. Kochevar, Phototox. Cancer Res., 48, 6794-6798 (1988).

4. S. Fickweiler, R.-M. Szeimies, and W. Baumler, Photochem. Photobiol. B: Biol., 38, 178-183 (1997).

5. S. Chatterjee, P. Davis, P. Gottschalk, M. Kurz, B. Sauervein, X. Yang, and G. Schuster, J. Am. Chem. Soc., 112, 6329-6338 (1990).

6. E. S. Voropai, M. P. Samtsov, K. N. Kaplevsky, A. A. Lugosky, and E. N. Alexandrova, Zh. Prikl. Spektrosk., 71, No. 2, 166-172 (2004).

7. N. A. Derevyanko, G. G. Dyadyusha, A. A. Ishchenko, and A. I. Tolmachev, Teor. Éksp. Khim., 19, 169-178 (1983).

8. V. I. Avdeeva and M. A. Al'perovich, Usp. Nauchn. Fotogr., 22, 84-90 (1984).

9. A. A. Ishchenko, Structure and Spectral-Luminescent Properties of Polymethine Dyes [in Russian], Naukova Dumka, Kiev (1994).

10. E. S. Voropai, M. P. Samtsov, and K. N. Kaplevsky, Zh. Prikl. Spektrosk., 70, No. 5, 635-641 (2003). 
11. A. J. Gordon and R. A. Ford, A Chemist's Companion, Wiley Interscience, New York (1972).

12. S. P. Velsko and R. G. Fleming, Chem. Phys., 65, 59-70 (1982).

13. V. Sundstrom and T. Gillbro, J. Chem. Phys., 86, 1788-1792 (1982).

14. E. S. Voropai, M. P. Samtsov, I. M. Gulis, D. V. Glushkov, K. N. Kaplevsky, A. E. Rad'ko, and K. A. Shevchenko, Spectral Instruments for Analytical Applications. Promising Developments [in Russian], E. S. Voropai, ed., Bel. Gos. Univ., Minsk (2005), 22-30.

15. G. O. Bekker, Introduction to Photochemistry of Organic Compounds [in Russian], Khimiya, Leningrad (1976).

16. A. A. Krasnovsky, Jr., Membr. Cell Biol., 12, 665-690 (1998).

17. E. S. Voropai and M. P. Samtsov, Opt. Spekstrosk., 82, 577-580 (1997).

18. T. A. Jenny and N. J. Turro, Tetrahedron Lett., 23, 2923-2926 (1982).

19. T. D. Poulsen, P. R. Ogilby, and K. V. Mikkelsen, J. Phys. Chem. A, 102, 9829 (1998).

20. A. P. Blokhin, M. F. Gelin, O. V. Buganov, V. A. Dubovsky, S. A. Tikhomirov, and G. B. Tolstorozhev, Zh. Prikl. Spektrosk., 70, No. 1, 66-72 (2003).

21. N. A. Borisevich, O. V. Buganov, S. A. Tikhomirov, G. B. Tolstorozhev, and G. L. Shkred, Kvantovaya Élektron. (Moscow), 28, 225-231 (1999).

22. S. P. McGlynn, T. Azumi, and M. Kinoshita, Molecular Spectroscopy of the Triplet State. Prentice-Hall International Series in Chemistry, Prentice-Hall, Englewood Cliffs, N. J. (1969).

23. V. A. Kuz'min, A. P. Darmanyan, V. I. Shirokova, M. A. Al'perovich, and I. I. Levkoev, Izv. Akad. Nauk SSSR, Ser. Khim., 3, 581-586 (1978).

24. E. S. Voropai and M. P. Samtsov, Opt. Spektrosk., 62, 64-67 (1987).

25. Y. H. Meyer, M. Pittman, and P. Plaza, J. Photochem. Photobiol. A: Chem., 114, 1-21 (1998).

26. I. Martini and G. V. Hartland, J. Phys. Chem., 100, 19764-19770 (1996).

27. I. P. Beletsky, ed., Ions and Ion Pairs in Organic Reactions [Russian translation], Mir, Moscow (1975). 